\title{
How do we handle traumatic pediatric carotid artery dissection?
}

\begin{abstract}
The authors present a case of a 3-year-old boy with a right temporoparietal lobe infarction that developed a day after a fall from height. In the following radiodiagnostic investigation, Magneticresonance (MR) angiography showed narrowing of the right cervical internal carotid artery (ICA) indicative of a cervical carotid dissection. With the aid of this case, the authors review the neuroimaging findings of traumatic carotid artery dissection and management of these cases in pediatric population.
\end{abstract}

Keywords: pediatric, traumatic, cervical, carotid artery, dissection
Volume 7 Issue 3 - 2017

\author{
Derya Burcu Hazer,' Övünç Erdem Çorapci, ${ }^{2}$ \\ Gökmen Reyhanli' \\ 'Mugla Sitki Kocman University, Faculty of Medicine, Turkey \\ ${ }^{2}$ Milas State Hospital, Turkey
}

Correspondence: Derya Burcu Hazer, Mugla Sitki

Kocman University, Faculty of Medicine, 48, Mugla, Turkey, Tel 902522 I |4838, Fax 9.02522E+ II,

Email burcuhazer@hotmail.com
Abbreviations: ICA, internal carotid artery; CAD, carotid artery dissection; MR, magnetic resonance; CT, computerized tomography

\section{Introduction}

Head and neck trauma in pediatric population differs from adult cases due to the immature cranio-cervical stability resulting from the weak neck musculature and large head to neck proportion. These cases also have drawn attention due to concomitant severe damage to the regional vascular structures such as carotid artery. ${ }^{1,2}$ In some cases, especially with skull base fractures and cervical vertebral column trauma; carotid artery dissections at the cervical segment can occur., The goal of our study is to draw attention to the radiodiagnostics and management of the traumatic pediatric cervical carotid artery dissection with the aid of a case of traumatic cervical carotid artery dissection (CAD).

\section{Case presentation}

A healthy 3-year-old boy fell from a second floor balcony. He cried immediately and vomited twice afterwards. A day after, he became inactive, so was taken to emergency department of our hospital. At the time of admission, his blood pressure was $113 / 58 \mathrm{~mm} \mathrm{Hg}$. Laboratory blood tests were normal. His neurological examination revealed left hemiparesis. There was no fever. The computed tomography (CT) showed intertabular occipital bone fracture that elongates to the right side (Figure 1A). He was hospitalized in intensive care unit. Magnetic resonance (MR) imaging showed an acute-stage infarction in the right temporoparietal lobe (Figure 1B,1C). Three-dimensional timeof-flight (3D-TOF) MR angiography revealed segmental narrowing of the right cervical Internal Carotid Artery (ICA) (Figure 1D). Thus, with the aid of neuro-imaging series, a traumatic dissection of the right cervical ICA, leading to cerebral infarction in the right temporoparietal lobe was detected. We have initiated intravenous anticoagulation treatment. After four days of treatment he had recovered totally in the upper extremities with slight paresis in the left leg. He was discharged from the intensive care unit, and his treatment was shifted to oral antiplatelet after two weeks. Three months later, the patient was completely free of symptoms, however control MR angiography revealed no recovery. Informed consent and the ethical permit was taken from the parents according to the local ethical committee guidelines.
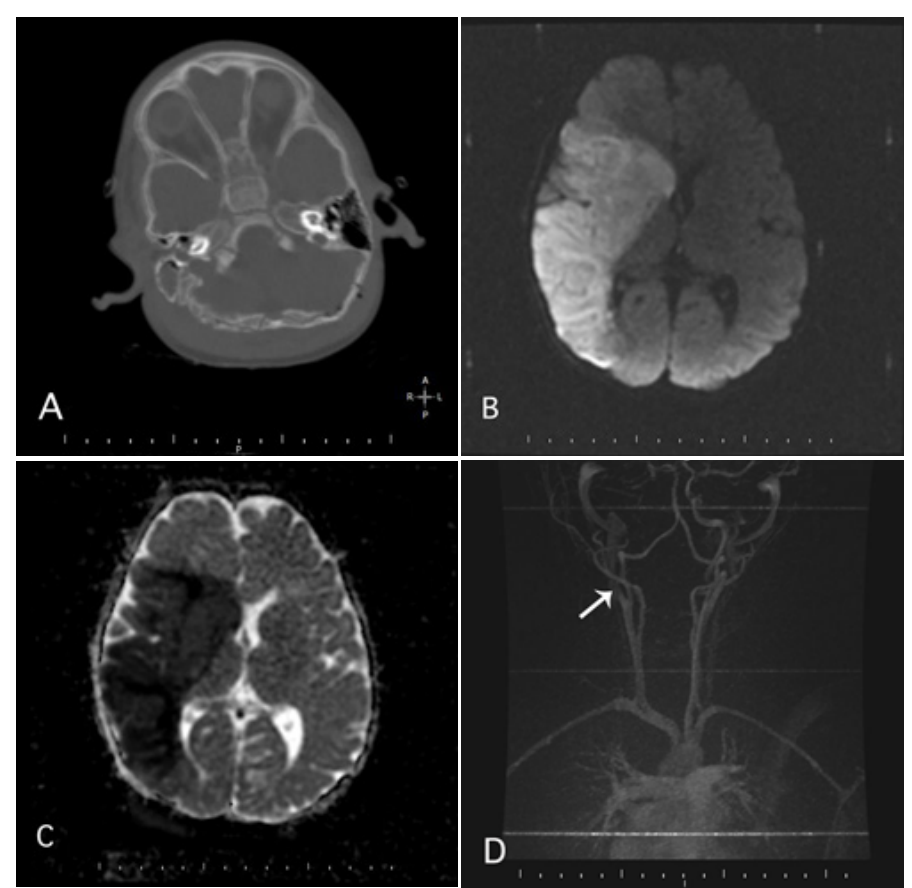

Figure I Radio diagnostic images of the case are presented.

(A) Cranial CT with bone enhancement at the time of admission is presented. Notice the occipital bone fracture that elongates to the right side. (B) Axial diffusion-weighted $M R$ image shows high signal intensity involving right temporopariatel region. (C) Corresponding ADC map of patient demonstrates restricted diffusion at the same area (D) Contrast enhanced 3D TOF MRA of patient demonstrates complete occlusion of the right ICA, at approximately $10 \mathrm{~mm}$ distal to the bifurcation (white arrow).

\section{Discussion}

Severe head and neck trauma in pediatric group has been a challenge for physicians if it is associated with CAD. ${ }^{5,6}$ In high velocity trauma, especially in paediatric population; upper cervical vertebral fractures with spinal cord injuries associated with severe head trauma can occur. Patients could be unconscious or revealing neurological deficits. On the contrary and any symptomatology of the artery dissection could be undetectable. Although proper treatment of the primary disease is achieved, patients could not recover fully due 
to misdiagnosed traumatic artery dissection. Therefore, it is crucial to diagnose arterial dissections at the admission and initialize the treatment accordingly.

In head and neck vasculature; the distal cervical ICA, V1, and V2 segments of the vertebral artery have the highest risk of traumatic dissection, as these are the most mobile segments within the cerebral vascular tree. ${ }^{7}$ It is stated that the most vulnerable segment of the carotid artery is the most distal cervical segment before entering the carotid canal at the skull base. The mechanism was explained as: the stretching of the artery at the proximal cervical vertebral column with hyperflexion or extension or rotation of the neck causes intimal tears in the artery wall. ${ }^{1,2,6}$ Subintimal tear in the arterial wall causes stenosis and thromboembolism; on the other hand, sub adventitial tear causes pseudo aneurysm formation and potential rupture. ${ }^{7}$ If either of these tears is able to reconnect to the true lumen, then a double lumen is created in the blood vessel, thereby causing a threat of thromboembolism as well. On the contrary, in adults; the dissection occurs usually at the bifurcation since atheromatous plaques are typically located there and may increase the risk for posttraumatic dissections. ${ }^{6}$ In our case, the dissection occurred at the $10 \mathrm{~mm}$ distal to the bifurcation, at the middle cervical segment of the internal carotid artery.

Neuroimaging plays an essential role in the diagnosis of CAD Cranial CT is the primary diagnostic tool for severe head trauma. It is able to show skull base fracture, which is commonly associated with traumatic CAD in children. ${ }^{8}$ Air extending along the carotid canal was shown to predict injury of the ICA with a sensitivity of $60 \%$ and specificity of $67 \% .{ }^{4}$ Head and neck CT angiography was reported to be as sensitive as MR Angiography in detecting CAD. ${ }^{9}$ However; its use in children is limited due to effect of ionizing radiation on the developing brain and radiosensitive structures like the eye lens. ${ }^{10} \mathrm{We}$ also with our case did not performed CT angiography due to these side effects. There was occipital bone fracture but the skull base was intact. Although there is cervical segment internal carotid artery dissection, there was no cervical spine fracture present either. The pathophysiology of the cervical CAD in our case is probably due to hypermobility of the neck with the head and stretching of the cervical segment of the artery. ${ }^{11,12}$

Conventional angiography is stated to be the diagnostic goldstandard in diagnosing $\mathrm{CAD}{ }^{3,6}$ There are specific angiographic findings in CAD: (1) identification of intimal flaps and double lumen, (2) aneurysmal pouch formation, (3) occlusion often tapering with a flame-shape appearance. ${ }^{13}$ However, similar to CT angiography; conventional angiography is not a choice of diagnostic tool in paediatric population. It is difficult to access vascular structures in children, and there is exposure to high doses of ionizing radiation. Therefore, MR and MR Angiography is the choice of diagnostic tool especially for the paediatric population. ${ }^{14}$

In MR and MR Angiography, there are several diagnostic findings presented in literature that points out pediatric traumatic CAD.${ }^{13}$ There may be absence of the normal flow void or altered luminal signal intensity and narrowing of the arterial lumen with hematoma within the arterial wall. ${ }^{13}$ Fat saturated, non-contrast T1-weighted MR images of both head and neck is used to detect the diagnostic intramural hematoma. MR Angiography also shows a tapered narrowing or occlusion of the dissected vessel. ${ }^{7}$ It is also helpful to determine the age of the thrombosis and therefore a guide to start the anticoagulation treatment or not. ${ }^{7}$ Our results also confirmed the high sensitivity of combined MRI and MRA in diagnosing paediatric traumatic CAD. We have also detected narrowing of the cervical carotid artery in TOF MR Angiography images. We were also able to detect the acute phase of the infarction and start the therapy immediately. However, MR Angiography gives limited information in cases with intracranial dissection and posterior circulation pathologies. Therefore; conventional angiography should be performed for such cases. ${ }^{6}$

The dilemma is still present for the algorithm of the treatment for children with CAD. The first step of the treatment is prevention of thromboembolism via antiplatelet and/or anticoagulation medications. It is recommended that antiplatelet agents can be administered in those patients with asymptomatic dissections, while anticoagulation therapy for those with symptomatic cerebral ischemia related to the CAD ${ }^{7}$. There is no consensus on the duration of the anticoagulation therapy, some administers for three months ${ }^{7}$; some for only 2 weeks. ${ }^{15,16}$ In our case, we have administered the anticoagulation therapy only for two weeks, because hemiparesis almost recovered within one week, and we continued with antiplatelet treatment for three months. In literature, it is recommended that for cases with prolonged symtomps or reinfarction despite of the treatment, interventional or microsurgical revascularization is the choice of treatment. ${ }^{17}$

\section{Conclusion}

Traumatic CAD is one of the most common "preventable" cause of paediatric acute cerebral infarction and may be associated with high morbidity and long-term neurological sequelae. Unfortunately, CAD is diagnosed after onset of focal neurological symptoms such as hemiparesis. Physicians should keep in mind the possibility of the traumatic CAD in children with head and neck trauma especially with history of acute acceleration/deceleration or rotational forces due to the unique biomechanical properties. One should suspect CAD if there is skull base fracture with air inclusions extending along the course of the carotid canal. Similarly, fat saturated, non-contrast T1-weighted MR images of both head and neck could be helpful to detect the intramural hematoma. Awareness of these findings should increase the detection rate of these potentially devastating vascular complications in paediatric head and neck trauma.

\section{Disclosures}

\section{Conflicts of interest}

None.

\section{Financial disclosure}

None.

\section{References}

1. Pinto PS, Meoded A, Poretti A, et al. The unique features of traumatic brain injury in children. Review of the characteristics of the pediatric skull and brain, mechanisms of trauma, patterns of injury, complications, and their imaging findings-part 2. J Neuroimaging. 2012;22(2):e18-e41.

2. Pinto PS, Poretti A, Meoded A, et al. The unique features of traumatic brain injury in children. Review of the characteristics of the pediatric skull and brain, mechanisms of trauma, patterns of injury, complications and their imaging findings-part 1. J Neuroimaging. 2012;22(2):e1-e17.

3. Rafay MF, Armstrong D, Deveber G, et al. Craniocervical arterial dissection in children: clinical and radiographic presentation. $J$ Child Neurol. 2006;21(1):8-16.

4. York G, Barboriak D, Petrella J, et al. Association of internal carotid artery injury with carotid canal fractures in patients with head trauma. AJR Am J Roentgenol. 2005;184(5):1672-1678.

5. de Borst GJ, Slieker MG, Monteiro LM, et al. Bilateral traumatic carotid artery dissection in a child. Pediatr Neurol. 2006;34(5):408-411. 
6. Orman G, Tekes A, Poretti A, et al. Posttraumatic Carotid Artery Dissection in Children: Not to be missed!. J Neuroimaging. 2014;24(5):467-472.

7. Pandey AS, Hill E, Al-Holou WN, et al. Management of pediatric craniocervical arterial dissections. Childs Nerv Syst. 2015;31(1):101-107.

8. Kopelman TR, Berardoni NE, O'Neill PJ, et al. Risk factors for blunt cerebrovascular injury in children: do they mimic those seen in adults? $J$ Trauma. 2011;71(3):559-564.

9. Robertson William C Jr, Given CA. Spontaneous intracranial arterial dissection in the young: diagnosis by CT angiography. BMC Neurol. 2006;6:16-21.

10. Brenner DJ, Hall EJ. Computed tomography — an increasing source of radiation exposure. $N$ Engl J Med. 2007;357(22):2277-2284.

11. Kindelberger D, Gilmore K, Catanese CA, et al. Hyperextension and rotation of head causing internal carotid artery laceration with basilar subarachnoid hematoma. J Forensic Sci. 2003; 48(6):1366-1368.

12. Rommel O, Niedeggen A, Tegenthoff M, et al. Carotid and vertebral artery injury following severe head or cervical spine trauma. Cerebrovasc Dis. 1999;9(4):202-209.
13. Chamoun RB, Mawad ME, Whitehead WE, et al. Extracranial traumatic carotid artery dissections in children: a review of current diagnosis and treatment options. J Neurosurg Pediatr. 2008; 2(2):101-108.

14. Lévy C, Laissy JP, Raveau V, et al. Carotid and vertebral artery dissections: three-dimensional time-of-flight MR angiography and MR imaging versus conventional angiography. Radiology . 1994;190(1): $97-$ 103.

15. Hughes KM, Collier B, Greene KA, et al. Traumatic carotid artery dissection: a significant incidental finding. Am Surg. 2000;66(11):1023-1027.

16. Powell RJ, Schermerhorn M, Nolan B, et al. Early results of carotid stent placement for treatment of extracranial carotid bifurcation occlusive disease. J Vasc Surg. 2004;39(6):1193-1139.

17. Selim M, Caplan LR. Carotid Artery Dissection. Curr Treat Options Cardiovasc Med. 2004;6(3):249-253. 